grows exponentially, and it rapidly becomes impractical to find the best solution. This problem is related to many optimization problems such as minimizing costs and maximizing efficiency.

Hopfield and Tank ${ }^{2}$ show that a suitably designed neural network can compute a good estimate of the best solution. When checked, it is not the best solution but it is quite close to the best and can be computed quickly. Their technique is to arrange the system so that only allowable solutions to the problem can occur. For example, if the city is visited eighth on the circuit, then no other city can also be visited eighth. When one city is chosen for that position on the trip, it inhibits all the other candidates for the eighth position. No city can be visited twice, so if a city is chosen for one position on the route, it inhibits its potential appearances in other positions on the route. Hopfield and Tank constructed the connection strengths and the energy function so that as the state of the network evolves, the states corresponding to routes with small total distance grow at the expense of other routes. If the initial state of the system is random, the final state of the system corresponds to a route with a small total distance and is an allowable route. If the computation is repeated a few times with different initial states, the best solution is close to the actual solution of the problem. For many practical applications, this is good enough to be satisfactory.

But special algorithms for this problem are able to get better and faster estimates, and DECtalk works. So why bother with NETtalk and its potential descendents? One reason is that these two quite different problems are solved with techniques that are basically that same: interconnected sets of simple elements coupled with modifiable connections. Special-purpose hardware built to make one compute quickly will make the other compute quickly as well. Also, the algorithms for a network computation are highly parallel, that is, all the elements are computing their outputs simultaneously. Network computations are therefore well suited for parallel computers. For example, optical hardware, with its high speed, low precision, parallelism and high interconnectivity, is an excellent match for the requirements of network computations. Several groups are now building special-

1. Sejnowski. T.J. \& Rosenberg, C.R. The Johns Hopkins University Electrical Engineering and Computer Science Technical Report JHUIEECS-86/0I (Baltimore. Maryland 1986).

2. Hopfield J J \& Tank, D. W. Biol Cybernet, 5, 141 (1985)

3. Rumelhart. D.E.. I linton. G.E. \& Williams, R.J. in Paral lel. Distributed Processing: Explorations in the Microstruc ture of Cognition (eds Rumelhart, D.E. \& McClelland. J.L.) (MIT. Cambridge, in the press)

4. Parker. D. MITTech Rep. TR-47(1085)

5. Yann Le Cun in Disordered Sustems and Biological Organization (eds Bienenstock. E. Fogelman Soulie. F. \& Weisbuch. G.) (Springer, Berlin, in the press).

6. Hopfield, J.J. Proc nam. Acad. Sci. U.S.A. 79, 2554 (1982)

7. Hopfield. J.J. Proc. nam. Acad. Sci. U.S. A. 81, $3088(1984$ purpose parallel hardware specifically for networks.

Once the hardware becomes available, it can be used for a wide range of problems, from optimization to learning rules for natural language, with only minor changes. Connectionist models may not give the best or fastest answer to any particular problem but will come up with a pretty good answer quickly. It is this potential flexibility and speed that makes practical applications so attractive. The brain may show similar computational flexibility and power for similar reasons.

James A. Anderson is in the Department of Psychology. Center for Neural Science and Center for Cognitive Science at Brown University, Providence, Rhode Island 02912, USA.

\title{
Physiology
}

\section{Chloride ions and cystic fibrosis}

\section{from Maynard Case}

Cystic fibrosis, a lethal inheritable disease, seems to involve defective transport of chloride ions across epithelia. Such a defect could be caused by an alteration in the number, characteristics or regulation of $\mathrm{Cl}$ channels in the plasma membrane of epithelial cells. Three recent articles ${ }^{1-3}$, one of which appears on page 467 of this issue, suggest it is the regulation of $\mathrm{Cl}^{-}$channel activity that is at fault.

The conventional electrophysiological studies on sweat gland ducts ${ }^{4}$ and airway epithelia ${ }^{5}$ (which are both affected in cystic fibrosis) point conclusively towards the involvement of decreased $\mathrm{Cl}^{-}$transport in the pathophysiology of this disease. The nature of this transport defect has now been studied independently by Welsh and Liedtke $\mathrm{e}^{1.2}$ and by Frizzell et al. ${ }^{3}$ using patch-clamp techniques.

The two groups have studied primary cultures of tracheal epithelial cells from normal subjects and cystic fibrosis patients and have recorded both from intact cells and excised, inside-out membrane patches. They both describe the presence of $\mathrm{Cl}$-selective channels on the apical membrane of these epithelial cells but under similar experimental conditions, Welsh and Liedtke observe only one type of channel whereas Frizzell et al. detect two channels. Also, the channel described by Welsh and Liedtke seems to be insensitive to $\mathrm{Ca}^{2+}$ but those described by Frizzell et al. are both $\mathrm{Ca}^{2+}$-sensitive.

Despite these apparent and important differences in the characteristics of the channels, which might perhaps reflect differences in tissue culture technique, the major findings of the two studies are similar. First, during recording from intact cells, $\mathrm{Cl}^{-}$-channel activity can be elicited by adrenaline or cyclic AMP in normal but not cystic fibrosis cells; and second, in excised patches, the biophysical properties and kinetic behaviour of the channels are identical in both cell types. This suggests that the problem in cystic fibrosis relates not to the presence or absence of the $\mathrm{Cl}^{-}$conductance but to the regulation of this conductance by a cyclic AMPdependent process.

Although both studies describe differ- ences between normal and diseased cells only about half the intact normal cells respond to stimulation by opening $\mathrm{Cl}^{-}$-channels whereas all excised patches from normal and diseased cells show activity. This may indicate active inhibition of the $\mathrm{Cl}^{-}$channel so that excising the patch or stimulating the cell removes some sort of brake. If so, perhaps the brake-release mechanism is at fault in cystic fibrosis. Alternatively, there could be two populations of epithelial cells in normal subjects, but only one in cystic fibrosis patients.

These articles refocus our attention on the pathway generating cyclic AMP, but as adrenaline causes the normal accumulation of cyclic AMP in cultured airway cells from cystic fibrosis patients, presumably the defect lies downstream from cyclic AMP generation itself. If a cyclic AMP-dependent pathway is involved in the disease, this may help to explain the effects of the disease on the pancreas - another prime target.

Secretion in the pancreas occurs across both acinar and ductal epithelia. Whereas the former is controlled by $\mathrm{Ca}^{2+}$-mobilizing agonists (cholecystokinin and acetylcholine), secretion across the ducts is controlled by secretin, which seems to act exclusively via cyclic AMP. Hence these studies on airway epithelia support the hypothesis that pancreatic ductal secretion is faulty in the disease. However, as cystic fibrosis is thought to be associated with defective $\mathrm{Cl}^{-}$secretion, it has always been a problem to explain why an epithelium lining the pancreatic ducts that secretes $\mathrm{HCO}_{3}^{-}$, rather than $\mathrm{Cl}^{-}$, should be affected (although this $\mathrm{HCO}_{3}^{-}$secretion is $\mathrm{Cl}^{-}$-dependent). If the fault lies in the regulation of the anion channel rather than the channel itself, as this recent work suggests, this objection is overcome.

Welsh, M.J. \& Liedthe, C.M. Nature 322, 467 (1986) Welsh MJ Science 232, 1648 (1986)

Frizzell. R.A., Rechkemmer, G. \& Shoemaker, R.L. Science 233. 558 (1986)

Quinton. P.M. Nature 301, 421 (1983)

Knowles M.R. Science 221, 1067 (1983)

Maynard Case is in the Department of Physiology, University of Manchester, Manchester $M 139 P T, U K$. 